\title{
Predictive value of orotate phosphoribosyltransferase in colorectal cancer patients receiving 5-FU-based chemotherapy
}

\author{
SHUJI KOMORI ${ }^{1,2}$, SHINJI OSADA ${ }^{1}$, HIROYUKI TOMITA ${ }^{3}$, KIMITOSHI NISHIO ${ }^{2}$, IWAO KUMAZAWA ${ }^{2}$, \\ SUSUMU TACHIBANA ${ }^{2}$, JUJI TSUCHIYA ${ }^{2}$ and KAZUHIRO YOSHIDA ${ }^{1}$ \\ ${ }^{1}$ Department of Surgical Oncology, Gifu University Graduate School of Medicine, Yanagido, Gifu 501-1194; \\ ${ }^{2}$ Department of Surgery, Ibi Welfare Hospital, Ibigawa, Gifu 501-0696; ${ }^{3}$ Department of Oncological Pathology, \\ Gifu University Graduate School of Medicine, Yanagido, Gifu 501-1194, Japan
}

Received July 26, 2012; Accepted January 9, 2013

DOI: $10.3892 / \mathrm{mco} .2013 .71$

\begin{abstract}
Pretreatment knowledge of chemosensitivity and side-effects of chemotherapy for colorectal cancer (CRC) patients are likely to ensure the best chemotherapeutic outcome. The aim of this study was to identify additional predictive factors of chemosensitivity to the key CRC treatment drug 5-fluorouracil (5-FU). Surgically obtained specimens from 106 patients treated for CRC were immunohistochemically assessed to investigate the correlation between the protein expression of the 5-FU metabolic enzymes orotate phosphoribosyltransferase (OPRT), thymidylate synthase (TS) and dihydropyrimidine dehydrogenase (DPD), and clinicopathological characteristics as well as the correlation between the protein expression and outcomes of 5-FU-based chemotherapy. A correlation was detected between the high expression of the 5-FU metabolic enzyme OPRT and negative lymph node metastasis $(\mathrm{P}=0.0496)$, as well as between DPD and advanced Tumor-Node-Metastasis (TNM) grade cases (IIIA-IVB) and positive lymph node metastases $(\mathrm{P}=0.0414$, respectively). In all 106 patients and in 79 patients undergoing 5-FU-based chemotherapy, survival was improved in those patients with a positive OPRT expression $(\mathrm{P}=0.0144$ and 0.0167 , respectively). OPRT expression was higher in the 79 patients with no recurrence $(\mathrm{P}=0.0179)$ as well as in patients treated with $\mathrm{R} 0$ surgery and 5-FU-based chemotherapy without side-effects $(\mathrm{P}=0.0126)$. Disease-free survival (DFS) rate was higher in patients without side-effects, and in patients with a positive OPRT expression without side-effects $(\mathrm{P}=0.0021$ and 0.0031 ,
\end{abstract}

Correspondence to: Dr Shinji Osada, Department of Surgical Oncology, Gifu University Graduate School of Medicine, 1-1 Yanagido, Yanagido, Gifu 501-1194, Japan

E-mail: sting@gifu-u.ac.jp

Abbreviations: 5-FU, 5-fluorouracil; DFS, disease-free survival; DPD, dihydropyrimidine dehydrogenase; MST, median survival time; OPRT, orotate phosphoribosyltransferase; OS, overall survival; SP, staining percentage; TS, thymidylate synthase

Key words: orotate phosphoribosyltransferase, 5-fluorouracil-based chemotherapy, prognosis, side-effect respectively). Findings of this study demonstrated that OPRT expression positively correlated with fewer side-effects of 5-FU-based chemotherapy and longer patient survival.

\section{Introduction}

Colorectal cancer (CRC) is one of the most common and life-threatening types of cancer worldwide (1). Through the development of multidisciplinary care and advances in surgical procedures and techniques over the past decade (2), additional treatment strategies and chemotherapeutic regimens have been found to be beneficial even for advanced-stage cancer. Improved combinations of 5-fluorouracil (5-FU)/folinic acid with irinotecan (FOLFIRI) or oxaliplatin (FOLFOX) have progressively increased tumor response up to $73 \%$, while the median survival time (MST) of patients with unresectable tumor has increased to 20 months (3).

Over the past several years, the selection of chemotherapeutic regimens has expanded greatly and satisfactorily due to the development of molecular-targeted therapy (4). In this context, 5-FU has become crucial in chemotherapeutic regimens for gastrointestinal cancer (5). 5-FU metabolic enzymes, orotate phosphoribosyltransferase (OPRT), thymidylate synthase (TS) and dihydropyrimidine dehydrogenase (DPD) are considered important predictive factors of the chemotherapeutic effect even in recently developed regimens (6-8). In the present study, the expression of these 5-FU metabolic enzymes and their correlation with beneficial outcomes, such as patient prognosis, as well as unfavorable outcomes, such as potential side-effects, were evaluated.

\section{Materials and methods}

Patients. Surgically obtained specimens from 106 CRC patients who were operated on between January, 2004 and April, 2008 in the Department of Surgical Oncology (Gifu University School of Medicine, Gifu, Japan), were investigated in this study (Table I). Patients comprised 54 males

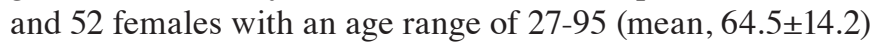
years. The disease stage was determined according to the pathological Tumor-Node-Metastasis (TNM) staging system [International Union Against Cancer (UICC), 7th edition]. 
Table I. Patient clinicopathological characteristics.

\begin{tabular}{l} 
Characterist \\
\hline Age (years) \\
$<60$ \\
$\geq 60$
\end{tabular}

Value (n)

Gender

Male

Female

Site

C-A-T

D-S-R

64

54

52

29

Differentiation

Well

Moderate

Poor

Depth of invasion

Mucosal-submucosal

Muscularis propria-serosa infiltrating (ai)

Lymphatic duct invasion

$\begin{array}{rr}0 & 9 \\ 1 & 52 \\ 2 & 29 \\ 3 & 16\end{array}$

Vascular invasion

0

1

2

3

All metastases

$(+)$

(-)

Liver

$(+)$

(-)

Lung

(+)

(-)

Peritoneal dissemination

$(+)$

(-)

Lymph node

$(+)$

$(-)$

Bone

$(+)$

(-)

Stage (pTNM)

0-IIC

IIIA-IVB
Table I. Continued.

\begin{tabular}{lc}
\hline Characteristics & Value (n) \\
\hline T (pTNM) & \\
Tis-T2 & 22 \\
T3-T4b & 84 \\
N (pTNM) & \\
N0 & 50 \\
N1-2 & 56 \\
M (pTNM) & \\
M0 & 83 \\
M1 (a/b) & 23 \\
\hline
\end{tabular}

Colorectal cancer staging ( $\mathrm{T}, \mathrm{N}, \mathrm{M}$ factors) was performed according to the pathological Tumor-Node-Metastasis staging system [International Union Against Cancer (UICC), 7th edition]. C-A-T, ceacum-ascending colon-transverse colon; D-S-R, descending colon-sigmoid colon-rectum; ai, tumor invasion of other organs and structures.

Pathological $\mathrm{T}$ indicators from Tis to $\mathrm{T} 2$ were observed in $20.8 \%$ of the patients. Distant metastases including overlapping metastases at each stage were detected at diagnosis in the lymph node, liver and lung. Peritoneal dissemination was detected in 17.0, 32.1, 22.6 and $8.4 \%$ of patients, respectively. As shown in Table II, 79 patients were treated with 5-FU-based chemotherapies, such as FOLFOX alone $(n=22)$ or in combination with bevacizumab $(n=3), 5-F U$ with leucovorin $(n=16)$, as well as uracil/tegafur (UFT) alone $(n=17)$ or in combination with leucovorin $(n=17)$. Side-effects of chemotherapy were classified according to the Common Terminology Criteria for Adverse Events (CTCAE v4.03). In this study, any event over grade 1 was regarded as a chemotherapy-induced side-effect. Experiments were planned carefully in accordance with the Ethical Standards of the Helsinki Declaration of 1975. In addition, the Institutional Research Ethics Board of the Gifu University (Gifu, Japan) approved this retrospective study.

Immunohistochemical staining. Formalin-fixed, paraffinembedded blocks of CRC specimens were obtained from the Division of Clinical Pathology of our institution, the Gifu University Graduate School of Medicine (Gifu, Japan). Details of the immunohistochemical staining techniques used have been previously described (9-11). Antigen retrieval was performed by autoclaving the sections in $0.01 \mathrm{M}$ citrate buffer for TS and in ethylenediaminetetraacetic acid (EDTA) (pH 8.0) buffer for DPD for $60 \mathrm{sec}$ at $120^{\circ} \mathrm{C}$ (DakoCytomation, Glostrup, Denmark). Endogenous peroxidase was quenched with $0.3 \%$ hydrogen peroxide methanol for $20 \mathrm{~min}$. The immunoperoxidase procedure [avidin-biotin-complex (ABC) method, VECTASTAIN ${ }^{\circledR}$ Elite ABC kit; Vector Laboratories, Inc., Burlingame, CA, USA] was used. Primary antibodies were incubated overnight at $4^{\circ} \mathrm{C}$. 3,3'-Diaminobenzidine tetrahydrochloride (DAB) (Dojin, Osaka, Japan) was applied for 2-3 min, and slides were counterstained with hematoxylin. Protein expression was evaluated by counting the number of cells with 
Table II. Number of patients receiving neoadjuvant chemotherapy.

5-FU-based chemotherapies (prior to treatment with FOLFOX, FOLFIRI)

5-FU-based chemotherapies (following treatment with FOLFOX, FOLFIRI)

Other chemotherapies No chemotherapies

\author{
Uracil/tegafur (UFT) alone \\ Uracil/tegafur (UFT) + oral leucovorin (Uzel) \\ Uracil/tegafur (UFT) + irinotecan (CPT-11) \\ Tegafur/gimeracil/oteracil potassium (TS-1) \\ 5 -FU + leucovorin \\ 5'-DFUR (Furtulon) \\ FOLFOX \\ FOLFOX + bevacizumab
}

Paclitaxel (Taxol)

None

17

17

1

1

16

2

54

22

3

25

1

26

5-FU, 5-fluorouracil; FOLFOX, 5-FU/folinic acid with oxaliplatin; FOLFIRI, 5-FU/folinic acid with irinotecan.
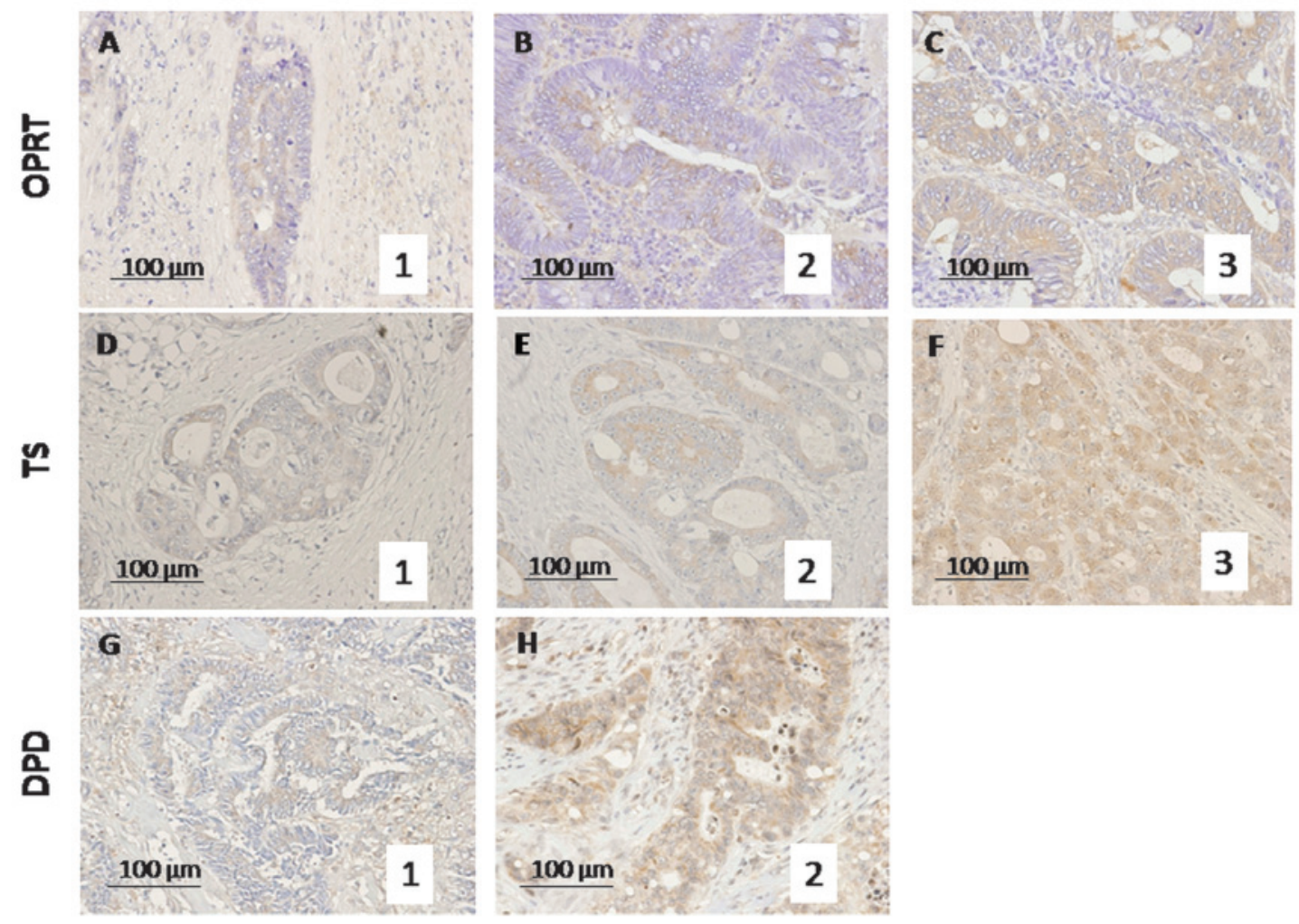

Figure 1. Photomicrographs showing scoring for evaluation of the expression of 5-FU metabolic enzymes. Expression of the 5-FU metabolic enzymes (A-C) OPRT, (D-F) TS and (G and H) DPD was evaluated on a 4-point scale based on the immunohistochemical staining percentage (SP): SP $<10 \%, 0$ points; $\mathrm{SP} \geq 10$ and $<30 \%, 1$ point; $\mathrm{SP} \geq 30$ and $<50 \%, 2$ points and $\mathrm{SP} \geq 50 \%, 3$ points. 5 -FU, 5 -fluorouracil; OPRT, orotate phosphoribosyltransferase; TS, thymidylate synthase; DPD, dihydropyrimidine dehydrogenase.

positive cytoplasmic staining among 200 tumor cells/field in 10 fields and scored as: staining percentage (SP) $<10 \%, 0$ points; $\mathrm{SP} \geq 10$ and $<30 \%, 1$ point; $\mathrm{SP} \geq 30$ and $50 \%, 2$ points and $\mathrm{SP} \geq 50 \%, 3$ points (Fig. 1 ), as previously reported $(7,12)$. The average score of each category was calculated. Two co-authors (HT and $\mathrm{KN}$ ) blinded to any prior knowledge of the clinico- pathological parameters evaluated the stained sections, and an expression index for each factor was calculated by averaging the percentage of positive cytoplasmic staining (7).

Statistical analysis. Data were presented as the mean \pm SD and were evaluated for statistical significance using the 
Table III. Correlation between clinicopathological characteristics and protein expression grade.

\begin{tabular}{|c|c|c|c|c|c|c|}
\hline Characteristics & $\mathrm{TS}$ & P-value & DPD & P-value & OPRT & P-value \\
\hline \multicolumn{7}{|l|}{ Age (years) } \\
\hline$<60$ & $0.50 \pm 0.70$ & & $0.07 \pm 0.34$ & & $1.36 \pm 1.13$ & \\
\hline$\geq 60$ & $0.47 \pm 0.77$ & 0.5647 & $0.09 \pm 0.29$ & 0.4050 & $1.41 \pm 1.03$ & 0.7529 \\
\hline \multicolumn{7}{|l|}{ Gender } \\
\hline Male & $0.50 \pm 0.79$ & & $0.07 \pm 0.26$ & & $1.44 \pm 1.07$ & \\
\hline Female & $0.46 \pm 0.69$ & 0.9940 & $0.10 \pm 0.35$ & 0.9340 & $1.33 \pm 1.07$ & 0.5686 \\
\hline \multicolumn{7}{|l|}{ Tumor location } \\
\hline C-A-T & $0.62 \pm 0.89$ & & $0.07 \pm 0.25$ & & $1.45 \pm 1.10$ & \\
\hline D-S-R & $0.43 \pm 0.67$ & 0.3796 & $0.09 \pm 0.33$ & 0.8648 & $1.36 \pm 1.06$ & 0.7381 \\
\hline \multicolumn{7}{|l|}{ Differentiation } \\
\hline Well & $0.46 \pm 0.73$ & & $0.09 \pm 0.34$ & & $1.19 \pm 1.02$ & \\
\hline Moderate & $0.46 \pm 0.68$ & & $0.09 \pm 0.28$ & & $1.63 \pm 1.09$ & \\
\hline Poor & $1.33 \pm 1.25$ & 0.3832 & $0.00 \pm 0.00$ & 0.8462 & $1.33 \pm 0.94$ & 0.1242 \\
\hline \multicolumn{7}{|c|}{ Depth of invasion } \\
\hline m-sm & $0.50 \pm 0.50$ & & $0.00 \pm 0.00$ & & $0.83 \pm 0.90$ & \\
\hline mp-si (ai) & $0.48 \pm 0.75$ & 0.6282 & $0.09 \pm 0.32$ & 0.4734 & $1.42 \pm 1.07$ & 0.1995 \\
\hline \multicolumn{7}{|c|}{ Lymphatic duct invasion } \\
\hline 0 & $0.44 \pm 0.50$ & & $0.00 \pm 0.00$ & & $1.11 \pm 0.87$ & \\
\hline 1 & $0.48 \pm 0.84$ & & $0.08 \pm 0.27$ & & $1.46 \pm 1.01$ & \\
\hline 2 & $0.52 \pm 0.68$ & & $0.14 \pm 0.43$ & & $1.55 \pm 1.07$ & \\
\hline 3 & $0.44 \pm 0.61$ & 0.6611 & $0.06 \pm 0.24$ & 0.4543 & $1.00 \pm 1.22$ & 0.5091 \\
\hline \multicolumn{7}{|c|}{ Vascular invasion } \\
\hline 0 & $0.36 \pm 0.69$ & & $0.00 \pm 0.00$ & & $1.16 \pm 0.97$ & \\
\hline 1 & $0.58 \pm 0.81$ & & $0.14 \pm 0.41$ & & $1.51 \pm 1.04$ & \\
\hline 2 & $0.46 \pm 0.63$ & & $0.12 \pm 0.32$ & & $1.50 \pm 1.15$ & \\
\hline 3 & $0.42 \pm 0.76$ & 0.7229 & $0.00 \pm 0.00$ & 0.3913 & $1.17 \pm 1.07$ & 0.6086 \\
\hline \multicolumn{7}{|l|}{ All metastases } \\
\hline$(+)$ & $0.38 \pm 0.63$ & & $0.06 \pm 0.24$ & & $1.18 \pm 1.09$ & \\
\hline$(-)$ & $0.57 \pm 0.82$ & 0.2319 & $0.11 \pm 0.36$ & 0.5568 & $1.57 \pm 1.02$ & 0.0545 \\
\hline \multicolumn{7}{|l|}{ Liver } \\
\hline$(+)$ & $0.44 \pm 0.69$ & & $0.09 \pm 0.28$ & & $1.15 \pm 1.09$ & \\
\hline$(-)$ & $0.50 \pm 0.76$ & 0.7013 & $0.08 \pm 0.32$ & 0.7505 & $1.50 \pm 1.04$ & 0.1065 \\
\hline \multicolumn{7}{|l|}{ Lung } \\
\hline$(+)$ & $0.33 \pm 0.47$ & & $0.04 \pm 0.20$ & & $1.21 \pm 1.08$ & \\
\hline$(-)$ & $0.52 \pm 0.80$ & 0.5210 & $0.10 \pm 0.34$ & 0.4731 & $1.44 \pm 1.06$ & 0.3639 \\
\hline \multicolumn{7}{|c|}{ Peritoneal dissemination } \\
\hline$(+)$ & $0.44 \pm 0.68$ & & $0.11 \pm 0.31$ & & $1.44 \pm 1.34$ & \\
\hline$(-)$ & $0.48 \pm 0.75$ & 0.8988 & $0.08 \pm 0.31$ & 0.6828 & $1.38 \pm 1.04$ & 0.9531 \\
\hline \multicolumn{7}{|l|}{ Lymph node } \\
\hline$(+)$ & $0.33 \pm 0.58$ & & $0.00 \pm 0.00$ & & $0.94 \pm 1.03$ & \\
\hline$(-)$ & $0.51 \pm 0.77$ & 0.4012 & $0.10 \pm 0.34$ & 0.1856 & $1.48 \pm 1.06$ & 0.0496 \\
\hline \multicolumn{7}{|l|}{ Bone } \\
\hline$(+)$ & $0.00 \pm 0.00$ & & $0.20 \pm 0.40$ & & $1.40 \pm 0.49$ & \\
\hline$(-)$ & $0.50 \pm 0.75$ & 0.0946 & $0.08 \pm 0.30$ & 0.2900 & $1.39 \pm 1.09$ & 0.8711 \\
\hline \multicolumn{7}{|l|}{ Stage (pTNM) } \\
\hline 0-IIC & $0.38 \pm 0.56$ & & $0.02 \pm 0.14$ & & $1.38 \pm 0.98$ & \\
\hline IIIA-IVB & $0.57 \pm 0.86$ & 0.4594 & $0.14 \pm 0.40$ & 0.0414 & $1.39 \pm 1.14$ & 0.9529 \\
\hline
\end{tabular}


Table III. Continued.

\begin{tabular}{|c|c|c|c|c|c|c|}
\hline Characteristics & $\mathrm{TS}$ & P-value & DPD & P-value & OPRT & P-value \\
\hline \multicolumn{7}{|l|}{ T (pTNM) } \\
\hline Tis-T2 & $0.50 \pm 0.58$ & & $0.05 \pm 0.21$ & & $1.32 \pm 1.02$ & \\
\hline T3-T4b & $0.48 \pm 0.78$ & 0.4646 & $0.10 \pm 0.33$ & 0.5457 & $1.40 \pm 1.08$ & 0.7497 \\
\hline \multicolumn{7}{|l|}{$\mathrm{N}(\mathrm{pTNM})$} \\
\hline N0 & $0.38 \pm 0.56$ & & $0.02 \pm 0.14$ & & $1.38 \pm 0.98$ & \\
\hline N1-2 & $0.57 \pm 0.86$ & 0.4594 & $0.14 \pm 0.40$ & 0.0414 & $1.39 \pm 1.14$ & 0.9529 \\
\hline \multicolumn{7}{|l|}{ M (pTNM) } \\
\hline M0 & $0.47 \pm 0.75$ & & $0.08 \pm 0.32$ & & $1.45 \pm 1.08$ & \\
\hline M1 (a/b) & $0.52 \pm 0.71$ & 0.6673 & $0.09 \pm 0.28$ & 0.8277 & $1.17 \pm 1.01$ & 0.3109 \\
\hline
\end{tabular}

A

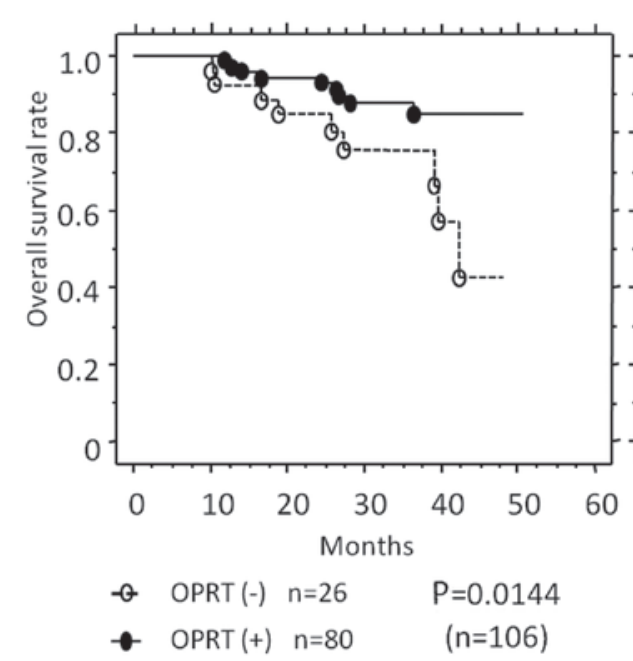

B

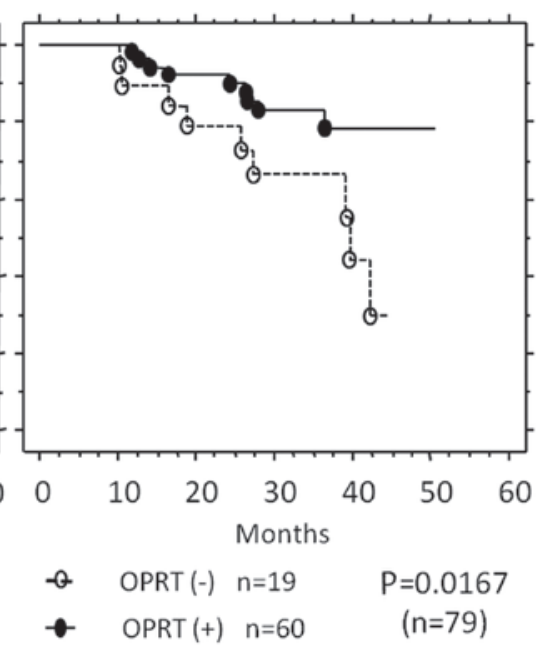

(A), MST, not reached vs. 42.3 months; $\mathrm{HR}=0.33 ; 95 \% \mathrm{Cl}, 0.1322-0.8403$

(B), MST, not reached vs. 39.5 months; $\mathrm{HR}=0.34 ; 95 \% \mathrm{Cl}, 0.1345-08584$

Figure 2. Kaplan-Meier curves showing the correlation between OPRT expression and median survival time (MST). (A) In the 106 patients, survival was improved in patients with a positive OPRT expression (closed circles, $n=80$ ) compared with patients with a negative expression (open circles, $\mathrm{n}=26$ ). (B) In 79 patients treated with 5-FU-based chemotherapy, survival was improved in patients with a positive OPRT expression (closed circles, $\mathrm{n}=60$ ) compared with patients with a negative expression (open circles, $\mathrm{n}=19$ ). OPRT, orotate phosphoribosyltransferase; HR, hazard ratio; CI, confidence interval; 5-FU, 5-fluorouracil..

Mann-Whitney $U$ and post-hoc tests (ANOVA). The Mann-Whitney U test was used to analyze the correlation between two factors in the clinicopathological data. Overall survival (OS), MST and disease-free survival (DFS) were calculated using the Kaplan-Meier method. The Cox proportional hazards model was used to compare these curves. $\mathrm{P}<0.05$ was considered to indicate a statistically significant difference. SPSS version 14 (SPSS, Inc., Chicago, IL, USA) and StatView 5.0 (SAS Institute Inc., Cary, NC, USA) were used for data management and analysis.

\section{Results}

Correlation between clinicopathological characteristics and protein expression grade. Positive immunohistochemical staining ( $\mathrm{SP} \geq 10 \%)$ was detected for OPRT in 80 (75.5\%), for TS in 38 (35.8\%) and for DPD in 8 (7.5\%) patients. Concerning the correlation with clinicopathological factors (Table III), the expression score of OPRT was lower in patients with positive lymph node metastasis compared with patients with a negative metastasis $(0.94 \pm 1.03$ vs. $1.48 \pm 1.06, \mathrm{P}=0.0496)$. Expression 
Table IV. Correlation between enzyme expression and side-effects.

Patients receiving 5-FU-based chemotherapy $(\mathrm{n}=79)$

Patients with no metastasis prior to surgery (receiving 5-FU-based chemotherapy) $(\mathrm{n}=49)$

\begin{tabular}{|c|c|c|c|c|c|c|}
\hline Enzymes & $\begin{array}{l}\text { Side-effects }(+) \\
\quad(n=43)\end{array}$ & $\begin{array}{l}\text { Side-effects (-) } \\
\quad(\mathrm{n}=36)\end{array}$ & P-value & $\begin{array}{l}\text { Side-effects }(+) \\
\quad(\mathrm{n}=20)\end{array}$ & $\begin{array}{c}\text { Side-effects }(-) \\
\quad(\mathrm{n}=29)\end{array}$ & P-value \\
\hline OPRT & $1.19 \pm 0.92$ & $1.64 \pm 1.18$ & 0.0908 & $1.05 \pm 0.86$ & $1.86 \pm 1.11$ & 0.0126 \\
\hline TS & $0.44 \pm 0.69$ & $0.53 \pm 0.87$ & 0.925 & $0.45 \pm 0.74$ & $0.52 \pm 0.86$ & 0.9323 \\
\hline DPD & $0.04 \pm 0.21$ & $0.17 \pm 0.44$ & 0.1473 & 0 & $0.17 \pm 0.46$ & 0.0865 \\
\hline
\end{tabular}

5-FU, 5-fluorouracil; OPRT, orotate phosphoribosyltransferase; TS, thymidylate synthase; DPD, dihydropyrimidine dehydrogenase.

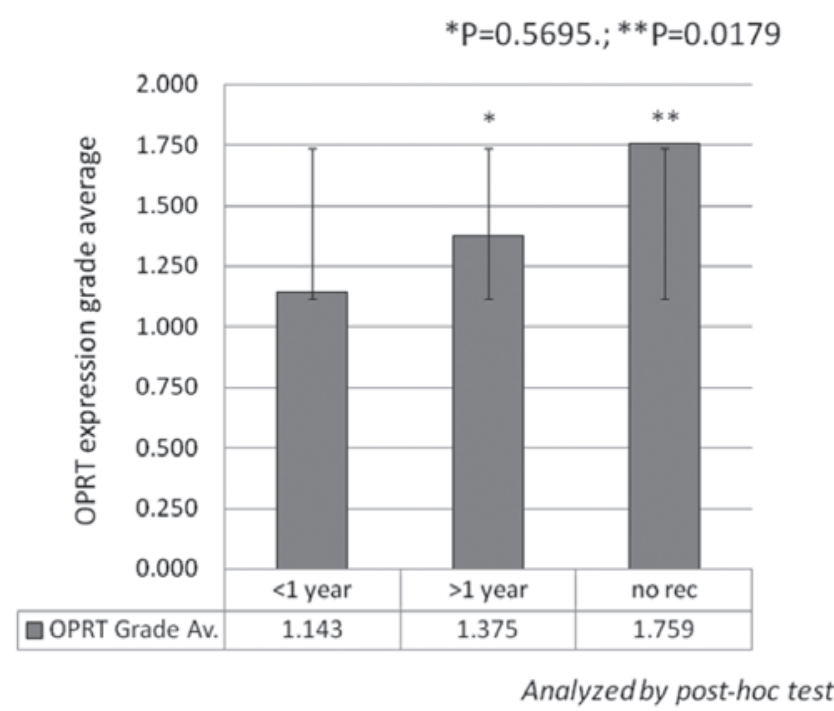

Figure 3. Correlation between OPRT expression and time to recurrence. In 79 patients treated with 5-FU-based chemotherapy, OPRT expression was higher in patients with no recurrence $(n=29)$ compared with those exhibiting early recurrence (recurrence within 1 year with any metastasis at surgery) $(n=42)$. No significant correlation was detected in 8 other patients with recurrence $>1$ year from surgery. OPRT, orotate phosphoribosyltransferase; 5-FU, 5-fluorouracil.

of DPD as detected by positive staining was markedly higher in both the advanced TNM stage (IIIA-IVB) vs. mild stage (0-IIC) lesions $(0.14 \pm 0.40$ vs. $0.02 \pm 0.14, \mathrm{P}=0.0414)$ and the positive vs. negative lymph node metastases for the $\mathrm{N}$ factor $(0.14 \pm 0.40$ vs. $0.02 \pm 0.14, \mathrm{P}=0.0414)$. No statistically significant differences were observed in other clinicopathological characteristics, such as age, gender, location of tumor, differentiation and depth of wall invasion.

Correlation between OPRT expression and MST, and time to recurrence. Expression of the three 5-FU metabolic enzymes was also evaluated from the viewpoint of patient prognosis (Fig. 2). In all the cases, the OS rate was higher in patients with a positive OPRT expression (75.5\%) vs. a negative OPRT expression $(24.5 \%)$ (MST, not reached vs. 42.3 months, $\mathrm{P}=0.0144$ ). In the 79 patients receiving 5-FU-based chemotherapy, the OS rate was also higher in patients with a positive OPRT expression (75.9\%) vs. a negative OPRT expression (24.1\%) (MST, not reached vs. 39.5 months, $\mathrm{P}=0.0167$ ). Furthermore, in these 79 patients, OPRT expression was markedly higher in the patients with no recurrence vs. those with early recurrence (recurrence within 1 year with any metastasis at surgery) $(1.759 \pm 0.951$ vs. $1.143 \pm 1.049, \mathrm{P}=0.0179)$ (Fig. 3). No statistically significant differences were detected in the expression of DPD and TS (data not shown).

Correlations between enzyme expression and side-effects, and between patient prognosis and side-effects. Side-effects were detected in 43/79 (54.4\%) patients receiving 5-FU-based chemotherapy. Of the 49 patients undergoing R0 surgery, side-effects occurred in $20(40.8 \%)$. The expression level of OPRT was markedly lower in patients positive for side-effects compared with patients negative for side-effects $(1.05 \pm 0.86$ vs. $1.86 \pm 1.11, \mathrm{P}=0.0126$ ) (Table IV). The correlation between side-effects and patient prognosis was compared in patients with no preoperative metastasis $(n=49)$. DFS was markedly better in patients without side-effects $(59.2 \%$, not reached) compared with those with side-effects $(40.8 \%, 13.6$ months) $(\mathrm{P}=0.0021)$ (Fig. 4A). Furthermore, in patients with positive OPRT expression and with no side-effects, DFS (not reached) was markedly improved compared with patients (not reached vs. 13.2 months, $\mathrm{P}=0.0031$; not reached vs. 8.2 months, $\mathrm{P}=0.0001$ ) (Fig. 4B). No statistically significant differences were detected in TS or DPD expression with regard to prognosis (data not shown).

\section{Discussion}

Combination chemotherapies with 5-FU/folinic acid have progressively improved tumor response and MST. According to the National Cancer Comprehensive Network (NCCN) Guidelines $^{\circledR}$ (Colon cancer, version 3, 2009; Rectal cancer, version 2, 2012), 5-FU is a key chemotherapeutic agent for CRC. However, the importance of 5-FU-related metabolic enzymes in the evaluation of patient outcome with respect to CRC patient prognosis remains controversial. In the present study, due to the fact that adjuvant chemotherapy for stage IIB/C CRC, considered a high-risk group for recurrence (13), is recommended but not required, patients were assessed according to TNM classification as IIIA-IVB patients with and 0 -IIC patients without chemotherapy. Cancer progression was observed to have a significant correlation with both OPRT and DPD expression, although not with TS expression. Furthermore, 
A

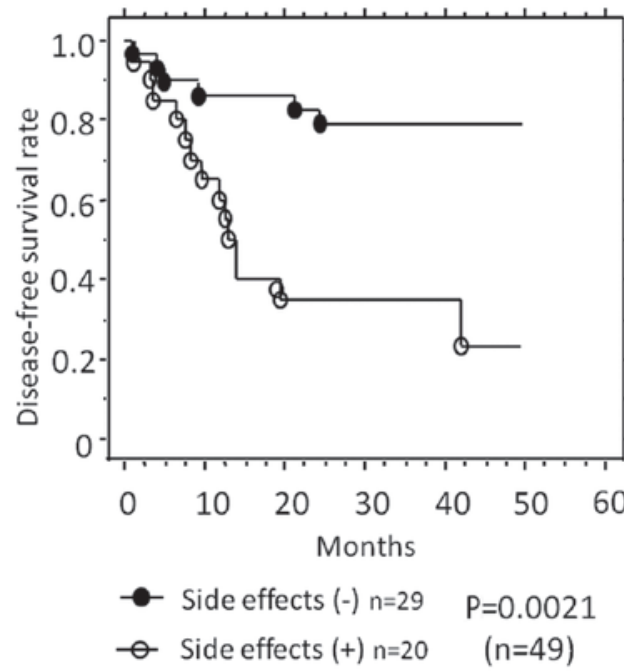

Median DFS, not reached vs 13.6 months, $\mathrm{HR}=0.22 ; 95 \% \mathrm{Cl}, 0.083-0.575$
B

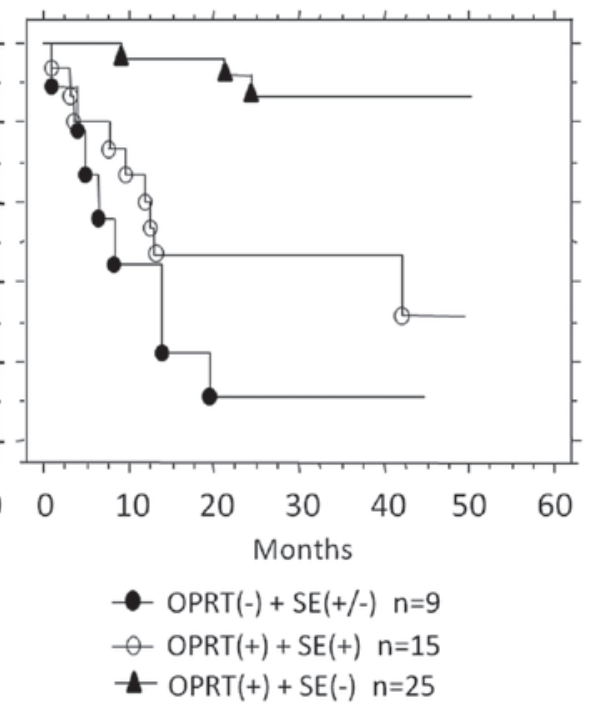

Figure 4. Kaplan-Meier curves showing patient prognosis with regard to side-effects. (A) Disease-free survival (DFS) after R0 surgery was improved in patients without (closed circles, $n=29$ ) compared with patients with side-effects (open circles, $n=20$ ). (B) DFS was improved in patients with a positive OPRT expression and no side-effects (triangles, $\mathrm{n}=25$ ) compared with patients either with a negative (closed circles, $\mathrm{n}=9$ ) or positive expression of OPRT, and with side-effects (open circle, $\mathrm{n}=15$ ). OPRT, orotate phosphoribosyltransferase; SE, side-effects; HR, hazard ratio; CI, confidence interval.

OPRT expression, but not TS and DPD expression, was identified as a predictor of patient prognosis and occurrence of side-effects caused by 5-FU-based chemotherapy. Of these three metabolic factors, the expression of OPRT protein was more easily detected in primary CRC with progressive depth of invasion or lymph node metastasis in gastrointestinal cancer $(12,14,15)$, even in the early stages of carcinogenesis $(16,17)$. Therefore, OPRT might be valuable as a predictive indicator of chemotherapeutic effects. The significance of TS or DPD expression has been controversial over the past decade $(18,19)$, and a recent study estimated TS to be critical for patient prognosis in pancreatic and breast cancers $(7,20)$. Furthermore, although DPD expression was associated with the progression of carcinogenesis in lymphatic/venous invasion in esophageal cancer or CRC (14,17), DPD itself has yet to be identified as a predictor of the effect of 5-FU-based chemotherapy.

OPRT is known to be one of the metabolic enzymes necessary for the conversion of 5-FU to its active nucleotide type, and several previous studies evaluated OPRT expression in mRNA $(6,21)$. The present study evaluated the expression of OPRT and has shown a correlation between OPRT protein expression and patient prognosis. Our results were based on the response to 5-FU as a cell-killing agent, and with regard to side-effects. When severe side-effects occur, chemotherapy should be discontinued, despite its effectiveness in inhibiting cancer growth. Previous studies of OPRT mRNA expression have demonstrated a correlation between the expression of this metabolic enzyme and the occurrence of side-effects in patients treated with 5-FU-based chemotherapy (22-24). According to an experimental study evaluating these factors (25), protein expression was more important as compared with mRNA expression in demonstrating enzyme activity. Thus, the present study suggests OPRT protein expression to be a predictor of the occurrence of side-effects.
Both 2-fluoro- $\beta$-alanine (F- $\beta$-alanine) and 5-fluoro-deoxyuridine diphosphate (FdUMP), metabolized by DPD and OPRT, respectively, were reported to induce neurological and gastrointestinal toxicity, as well as hematotoxicity (26-28). It is reasonable to expect that a decrease in the dose of F- $\beta$-alanine, which induces neurological toxicity, results in a reduction of side-effects. However, FdUMP has two opposing effects: first, as an anticancer agent, and second, as a factor in gastrointestinal toxicity and hematotoxicity, although an anticancer drug with few side-effects as well as increased carcinostatic effects is ideal for cancer patients. Consequently, future studies verifying the mechanism of FdUMP and its interactions with other factors in humans are required.

In conclusion, OPRT expression was found to be a predictive factor of patient prognosis and occurrence of side-effects in CRC patients administered 5-FU-based chemotherapy. Following the detailed investigation of specimens resected surgically or by endoscopic biopsy, clinicians may be able to determine the most effective chemotherapeutic regimen.

\section{Acknowledgements}

The authors would like to thank the Taiho Pharmaceutical Co., Ltd. (Tokyo, Japan) for kindly providing the OPRT, TS and DPD antibodies.

\section{References}

1. Siegel R, Naishadham D and Jemal A: Cancer statistics. CA Cancer J Clin 62: 10-29, 2012.

2. Osada S, Imai H, Sasaki Y, et al: Strategy for synchronous and multiple liver metastasis. Hepatogastroenterology 59: 198-203, 2012.

3. de Gramont A, Figer A, Seymour M, et al: Leucovorin and fluorouracil with or without oxaliplatin as first-line treatment in advanced colorectal cancer. J Clin Oncol 18: 2938-2947, 2000. 
4. Giantonio BJ, Catalano PJ, Meropol NJ, et al: Bevacizumab in combination with oxaliplatin, fluorouracil, and leucovorin (FOLFOX4) for previously treated metastatic colorectal cancer: results from the Eastern Cooperative Oncology Group Study E3200. J Clin Oncol 25: 1539-1544, 2007.

5. Hamilton SR: Targeted therapy of cancer: new roles for pathologists in colorectal cancer. Mod Pathol 21: S23-S30, 2008.

6. Yamada $\mathrm{H}$, Iinuma $\mathrm{H}$ and Watanabe $\mathrm{T}$ : Prognostic value of 5-fluorouracil metabolic enzyme genes in Dukes' stage B and C colorectal cancer patients treated with oral 5-fluorouracil-based adjuvant chemotherapy. Oncol Rep 19: 729-735, 2008.

7. Komori S, Osada S, Mori R, et al: Contribution of thymidylate synthase to gemcitabine therapy for advanced pancreatic cancer. Pancreas 39: 1284-1292, 2010.

8. Komori S, Osada S and Yoshida K: Novel strategy with gemcitabine for advanced pancreatic cancer. ISRN Oncol 2011: 936893, 2011.

9. Tomita H, Yamada Y, Oyama T, et al: Development of gastric tumors in $\mathrm{Apc}(\mathrm{Min} /+)$ mice by the activation of the betacatenin/Tcf signaling pathway. Cancer Res 67: 4079-4087, 2007.

10. Osada S, Saji S and Kuno T: Clinical significance of combination study of apoptotic factors and proliferating cell nuclear antigen in estimating the prognosis of hepatocellular carcinoma. J Surg Oncol 85: 48-54, 2004

11. Osada S, Saji S and Takahashi T: A case report of papilla Vater carcinoma showing positive expression of thymidine phosphorylase. Hepatogastroenterology 51: 375-377, 2004.

12. Kawahara A, Akagi Y, Hattori S, et al: Higher expression of deoxyuridine triphosphatase (dUTPase) may predict the metastasis potential of colorectal cancer. J Clin Pathol 62: 364-369, 2009.

13. Van Cutsem EJ and Oliveira J: Colon cancer: ESMO clinical recommendations for diagnosis, adjuvant treatment and follow-up. Ann Oncol 19: ii29-ii30, 2008.

14. Tokunaga Y, Sasaki H and Saito T: Clinical role of orotate phosphoribosyl transferase and dihydropyrimidine dehydrogenase in colorectal cancer treated with postoperative fluoropyrimidine. Surgery 141: 346-353, 2007.

15. Ochiai T, Sugitani M, Nishimura K, et al: Impact of orotate phosphoribosyl transferase activity as a predictor of lymph node metastasis in gastric cancer. Oncol Rep 14: 987-992, 2005.

16. Sanada Y, Yoshida K, Hihara J and Okada M: Expression of orotate phosphoribosyltransferase in colorectal carcinoma: An immunohistochemical analysis in several components of neoplastic lesions. Oncol Rep 20: 1005-1011, 2008.

17. Tsutani Y, Yoshida K, Sanada Y, et al: Dihydropyrimidine dehydrogenase and orotate phosphoribosyltransferase in esophageal cancer patients: Correlation with clinicopathological factors and prognosis. Mol Med Rep 1: 713-719, 2008.
18. Ichikawa W, Uetake H, Shirota Y, et al: Combination of dihydropyrimidine dehydrogenase and thymidylate synthase gene expressions in primary tumors as predictive parameters for the efficacy of fluoropyrimidine-based chemotherapy for metastatic colorectal cancer. Clin Cancer Res 9: 786-791, 2003.

19. Ichikawa W, Uetake H, Shirota Y, et al: Both gene expression for orotate phosphoribosyltransferase and its ratio to dihydropyrimidine dehydrogenase influence outcome following fluoropyrimidine-based chemotherapy for metastatic colorectal cancer. Br J Cancer 89: 1486-1492, 2003.

20. Hosono Y, Osada S, Nawa M, et al: Combination therapy of 5 -fluorouracil with rapamycin for hormone receptor-negative human breast cancer. Anticancer Res 30: 2625-2630, 2010.

21. Ochiai T, Nishimura K, Noguchi H, et al: Prognostic impact of orotate phosphoribosyl transferase among 5-fluorouracil metabolic enzymes in resectable colorectal cancers treated by oral 5-fluorouracil-based adjuvant chemotherapy. Int J Cancer 118: 3084-3088, 2006.

22. Lecomte T, Ferraz JM, Zinzindohoue F, et al: Thymidylate synthase gene polymorphism predicts toxicity in colorectal cancer patients receiving 5-fluorouracil-based chemotherapy. Clin Cancer Res 10: 5880-5888, 2004.

23. Ichikawa W, Takahashi T, Suto K, Sasaki Y and Hirayama R Orotate phosphoribosyltransferase gene polymorphism predicts toxicity in patients treated with bolus 5-fluorouracil regimen. Clin Cancer Res 12: 3928-3934, 2006.

24. van Kuilenburg AB: Dihydropyrimidine dehydrogenase and the efficacy and toxicity of 5-fluorouracil. Eur J Cancer 40: 939-950, 2004.

25. Tsuchida M, Yamato Y, Hashimoto T, et al: Expression of 5-fluorouracil-related enzymes in lung cancer: ELISA characterizes enzyme activity and messenger RNA expression. Oncol Rep 21: 1037-1043, 2009.

26. Arellano M, Malet-Martino M, Martino R and Spector T: 5-Ethynyluracil (GW776): effects on the formation of the toxic catabolites of 5-fluorouracil, fluoroacetate and fluorohydroxypropionic acid in the isolated perfused rat liver model. Br J Cancer 76: 1170-1180, 1997

27. Yoshisue K, Hironaga K, Yamaguchi S, Yamamoto A, Nagayama S and Kawaguchi Y: Reduction of 5-fluorouracil (5-FU) gastrointestinal (GI) toxicity resulting from the protection of thymidylate synthase (TS) in GI tissue by repeated simultaneous administration of potassium oxonate (Oxo) in rats. Cancer Chemother Pharmacol 46: 51-56, 2000.

28. Kato T, Shimamoto Y, Uchida J, et al: Possible regulation of 5-fluorouracil-induced neuro- and oral toxicities by two biochemical modulators consisting of S-1, a new oral formulation of 5-fluorouracil. Anticancer Res 21: 1705-1712, 2001. 